\title{
Monitoring SEU Parameters at Reduced Bias
}

\author{
D.R. Roth, P.J. McNulty, W.G. Abdel-Kader, and L. Strauss \\ Department of Physics and Astronomy \\ Clemson University \\ Clemson, SC 29634-1911 \\ E.G. Stassinopoulos \\ NASA Goddard Spaceflight Center \\ Greenbelt, MD 20771
}

\begin{abstract}
SEU sensitivity of a CMOS SRAM increases with decreasing bias in such a manner that the critical charge exhibits a linear dependence on bias. This should allow proton and neutron monitoring of SEU parameters even for radiation hardened devices. The sensitivity of SEU rates to the thickness of the sensitive volume is demonstrated and procedures for determining the SEU parameters using protons are outlined.

\section{Introduction}

The SEU sensitivity of devices is known to increasing with decreasing bias applied to the device. According to the FirstOrder Model of SEUs (1), the change in sensitivity can be due to changes in the dimensions of the sensitive volume, to changes in the critical charge required to upset the device, or both. Earlier studies in our laboratory have shown that there is only a weak dependence of the thickness of the sensitive volume on the bias, and the lateral dimensions should not vary significantly either (1). Changes in SEU sensitivity with bias should, therefore, be dominated by changes in the critical charge. If manufacturing variations result in the device becoming more SEU sensitive at normal bias, there should be a corresponding change in the devices sensitivity at reduced bias. This has potential for an inexpensive procedure for monitoring the SEU hardness of devices manufactured under QML protocol because one might be able to take advantage of the increased sensitivity to use radioactive sources such as neutron and alpha sources for monitoring in the manufacturing site.
\end{abstract}

This study is part of a systematic study of SEE sensitivity designed to improve calculations of SEE rates. This includes improved determination of SEU parameters. Additional long-term goals are to simplify testing procedures and to develop a method to monitor SEU sensitivity on a continuous basis using radioactive sources. It is also apparent that proton and neutron induced upsets are of more concern for some devices and environments than heavy ions, and it would be highly desirable to develop a procedure for obtaining all the SEU parameters from proton testing alone rather than attempt to extrapolate the proton behavior from heavy ion testing.
This paper describes some preliminary data on SEU testing at reduced bias and a new simple procedure for determining the thickness of the sensitive volume using energetic protons. The proton analysis provides a mechanism explaining the angular asymmetry observed for some devices by most testing groups.

SEU Testing at Reduce Bias

A systematic study of this relationship has begun in this laboratory using a CMOS SRAM, as a test vehicle. Figure 1 is a schematic of the test hardware used in the study. The memory was loaded with a test pattern $\mu$ nder normal bias and it was then disconnected from the CPU before the bias was reduced and the irradiation begun. The protocol for testing SRAMS using low-voltage retention modes is as follows:
1. Load test pattern into the SRAM.
2. Electrically isolate the SRAM.
3. Reduce the bias on the SRAM (Keep CE at Vdd).
4. Irradiate SRAM (Keep CE at Vdd).
5. Raise the bias on the SRAM (Keep CE at Vdd).
6. Electrically reconnect the SRAM (Keep CE at Vdd).
7. Read SRAM bit pattern.
8. Compare the before and after patterns for upsets.

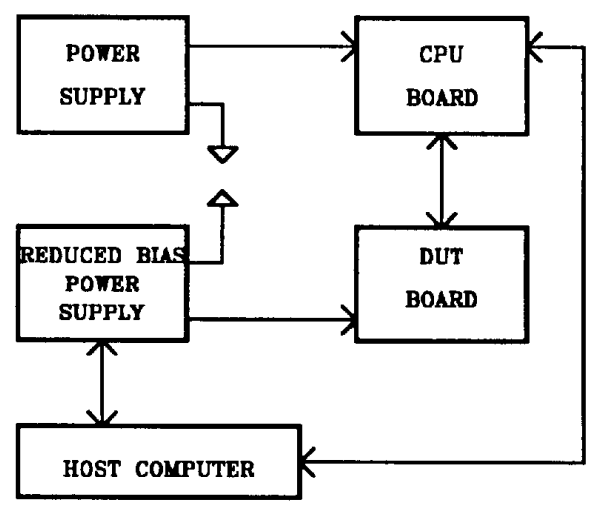

Fig. 1. Schematic of the test hardware used in the irradiations. 
The exposures were carried out at the Tandem Van de Graaff facility at Brookhaven National Laboratory. Irradiations were with different ion species at various angles of incidence.

The SEU response curves obtained at different values of the bias are shown in Fig. 2. The plots are of the cross section corrected to its equivalent value at normal incidence by multiplying the measured value by the square of the secant of the angle of incidence $\theta$ versus the value of the effective LET (LET $\sec ^{2} \theta$ ). The choice of these correction factors are determined by the analysis of charge collection presented earlier and in this issue and is consistent with the cord-length distribution in sensitive volumes found in modern SRAMs (2-6). The threshold LET was taken to be the value of the effective LET where the cross section reached $50 \%$ of its value at the beginning of the plateau region. This use of $50 \%$ threshold is different than the values of $10 \%$ and $25 \%$ traditionally used to predict SEU rates. These lower values are normally used to produce conservative estimates with "Figure of Merit" type formulas (7). The value of threshold at $50 \%$ corresponds to the value of LET where an ion strike has $50 \%$ probability of inducing an upset and is, therefore, a better representation of threshold for comparing the SEU potential of different radiation types. The data taken at a bias of $2.5 \mathrm{~V}$ was used to determine the beginning of the plateau. The horizontal line in Fig. 2 represents the cross section at $50 \%$ of the plateau value. The conclusions of this paper are not sensitive to whether we choose a cross section at $50 \%$ or $25 \%$ of plateau for the threshold LET.

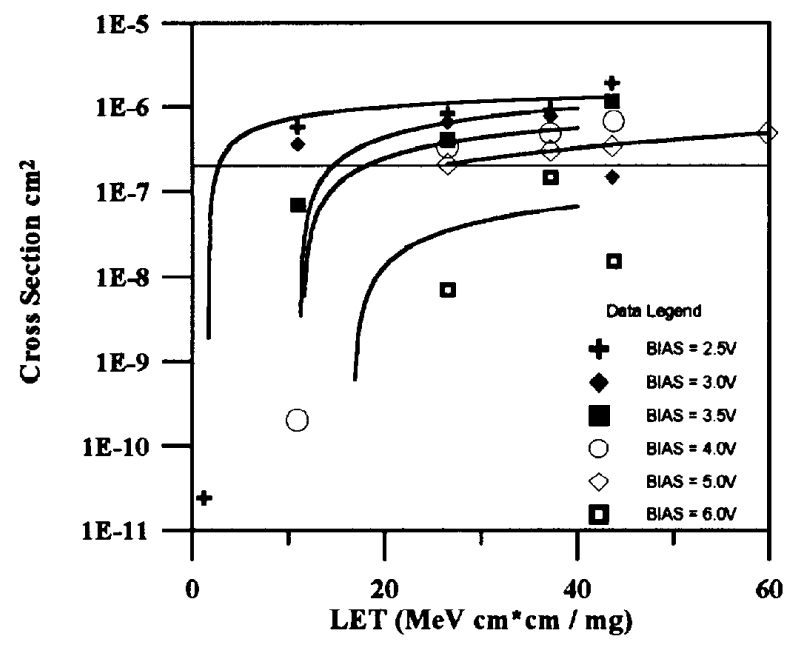

Fig. 2. SEU cross section corrected to normal incidence versus the effective LET of the incident ions.

The value of the critical charge obtained from the LET corresponding to $50 \%$ of plateau (Threshold LET) is plotted versus the applied bias in Fig. 3. The relationship is linear. This means that the value corresponding to $5 \mathrm{~V}$ or any intermediate operating voltage can be obtained by extrapolation. If the threshold LET (or the critical charge) can be shown to vary with bias in a regular way, then changes in a device's SEU sensitivity can be monitored at any bias since changes in manufacturing that impact threshold at the operating voltage will change the threshold at other voltages as well.

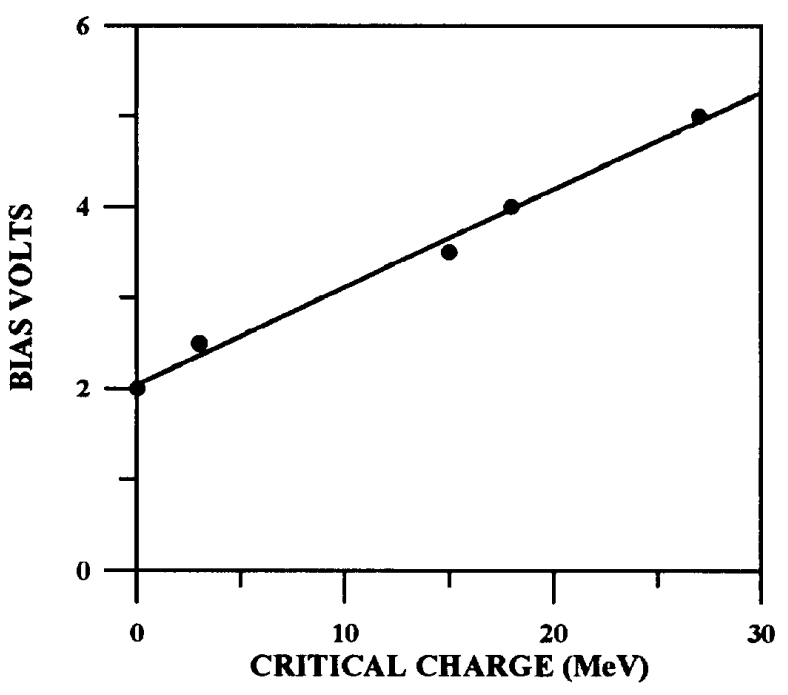

Fig. 3. Threshold versus applied bias.

\section{Determining SEU Parameters}

The remaining SEU parameters are the dimensions of the sensitive volume, particularly the thickness. The SEUsensitive structure is typically the n-channel drain whose lateral dimensions can be obtained from the mask. Uncertainty in the thickness results in significant uncertainties in the predicted SEU rates. Procedures for using charge collection suitable for use with an alpha source to determine the dimensions of the sensitive volume have been described earlier $(1,4)$. Measurements with test structures show that the area of the sensitive volume is insensitive to bias, and the thickness increases about $20 \%$ from zero to full bias. This small change in peak position in the charge collection means that reasonable estimates of the thickness of the sensitive volume can be made at any bias between zero and full bias and then corrected to the bias value used in operation.

Recent studies with protons (5) have shown that a series of measurements of the SEU cross section when the device is exposed to protons at different energies and angles of incidence is sufficient to confirm the dimensions of the sensitive volume and to determine the value of the critical charge. This CMOS SRAM, like all SEU-hard parts, is immune to protons when the exposures are carried out at bias values within the device's normal operating range. However, reducing the bias sufficiently makes even SEU-hardened parts proton sensitive. Measurements of proton SEU cross sections 
are much less expensive because they do not involve vacuum systems and the beam energy can be changed with degraders. The results of CUPID simulations for an SRAM device exposed to $148 \mathrm{MeV}$ protons at normal and grazing incidence are shown in Fig. 4.

The simulations in Fig. 4a were carried out assuming the sensitive volume was $1 \mu \mathrm{m}$ thick. The measured SEU cross sections are represented by horizontal lines terminating on the appropriate simulation curve. Vertical lines from the termination point of the cross section on the curve mark the value the critical charge would have to be for that simulation to be correct. According to the First Order Model, only one set of SEU parameters should fit measurements at all angles. Figure $4 \mathrm{~b}$ shows the same analysis for a thickness of $12 \mu \mathrm{m}$. Here the curves for normal and grazing incidence overlay and the difference in values of the critical charge $(\sim 0.1 \mathrm{MeV})$ are not resolvable in the figure indicating that the correct thickness is much closer to $12 \mu \mathrm{m}$ than $1 \mu \mathrm{m}$.

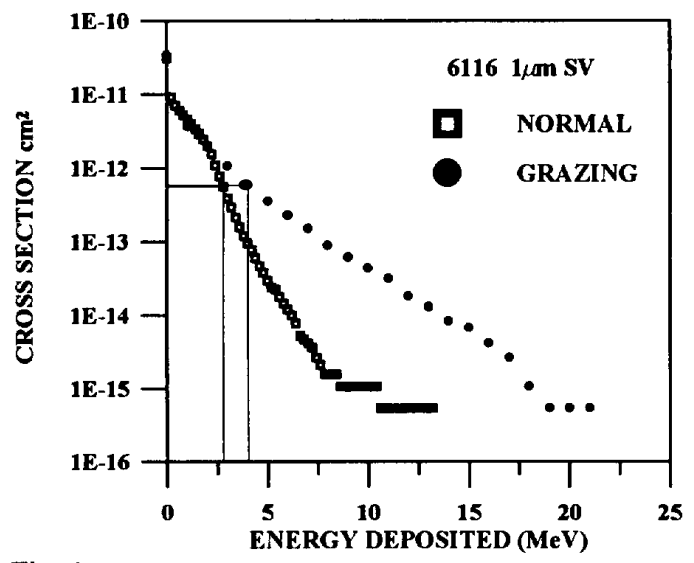

Fig. 4 a.

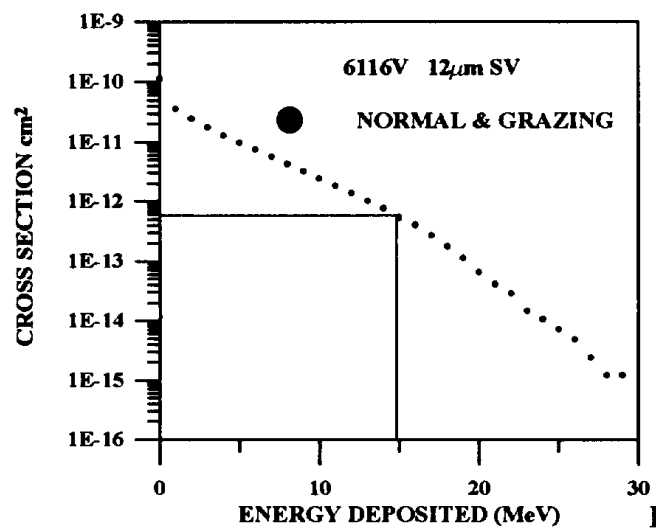

Fig. 4b.

Fig. 4. Cross section per bit for depositing at least energy $E$ versus E. Calculations are carried out for $148 \mathrm{MeV}$ protons incident at normal incidence. The thickness is a) $t=l \mu \mathrm{m}$ and b) $\mathrm{t}=12 \mu \mathrm{m}$.
Figure 5 plots the magnitude of the difference between the values of the critical charge obtained for normal and grazing incidence versus the value of the thickness. The minimum is at about $13 \mu \mathrm{m}$ in agreement with an earlier determination using charge collection measurements (6). This method has been applied to single event latch-up (8) as well as SEU. Table 1 compares the values of the thickness of the sensitive volumes determined for SEU and SEL by this method with the values obtained by charge collection spectra (CCS) measurements, Scanning Electron Microscope (SEM) measurements or the thickness of the epi-layer. We have found that the epi-layer plus $1 \mu \mathrm{m}$ represents an effective upper limit to the depth of charge collection. For thin epilayers it is a reasonable estimate of the thickness of the sensitive volume. The agreement is quite good in all cases. Once the thickness of the sensitive volume is resolved, the value of the threshold energy deposition (critical charge) can be obtained from the appropriate simulation run. A reasonable approximation for the threshold energy deposition can be obtained from Fig. $4 b$.

\begin{tabular}{|l|c|c|c|c|}
\hline DEVICE & $\begin{array}{c}\text { CCS } \\
(\mu \mathrm{m})\end{array}$ & $\begin{array}{c}\text { PROTON } \\
(\mu \mathrm{m})\end{array}$ & $\begin{array}{c}\text { EPI+1 } \\
(\mu \mathrm{m})\end{array}$ & $\begin{array}{c}\text { SEM } \\
(\mu \mathrm{m})\end{array}$ \\
\hline IDT6116 & 13.6 & 13.1 & & \\
\hline NEC4464 & & 6.7 & & 7.1 \\
\hline HC 6364 & 5.0 & 5.3 & & \\
\hline AMD93L422 & $*$ & 3.1 & 3.0 & \\
\hline
\end{tabular}

TABLE 1. THICKNESS

* Not available because of the amplification of the charge collection signal in bipolar parts makes thickness estimates impossible.

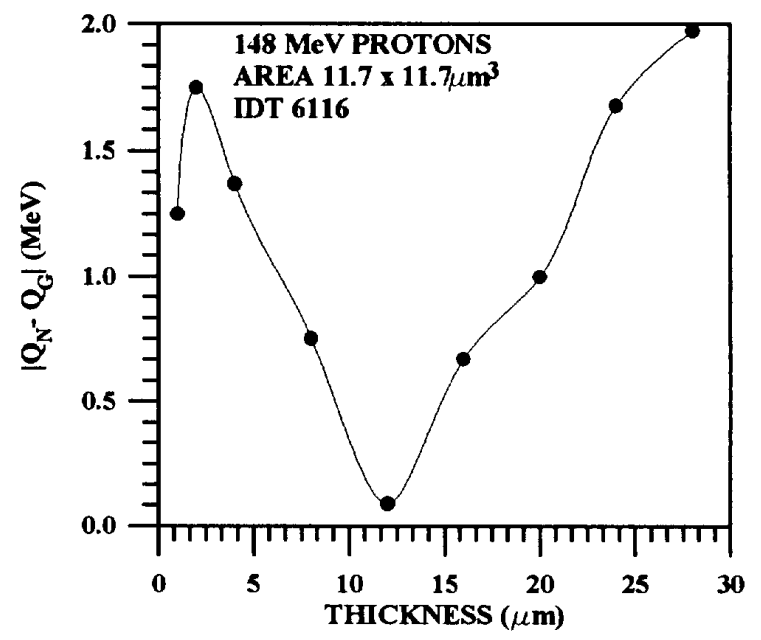

Fig. 5. Magnitude of the difference between the values of the critical charge determine for normal and grazing incidence plotted versus the thickness of the sensitive volume. The minimum is at $13 \mu \mathrm{m}$. 
Predicting Threshold LET

If one has determined the thickness $t$ of the sensitive volume and the value of the threshold energy that must be deposited for an upset, $Q_{c}$, the threshold LET to be expected for heavy ions at normal incidence can be predicted in $\mathrm{MeV} \mathrm{cm} / 2 / \mathrm{mg}$ from the following:

$$
\text { LET }=Q_{c} \rho / t
$$

where $Q_{c}$ is in $\mathrm{MeV}, \rho$ the density is in $\mathrm{mg} / \mathrm{cm}^{3}$, and the thickness of the sensitive volume is in $\mathrm{cm}$. The values predicted are compared to estimates based on heavy-ion data in Table 2, The values of threshold LET correspond to the value for $50 \%$ of the plateau value rather than the onset threshold.

\begin{tabular}{|l|l|l|l|}
\hline DEVICE & $\begin{array}{l}\text { CUPID } \\
(\mathrm{MeV}- \\
\left.\mathrm{cm}^{2} / \mathrm{mg}\right)\end{array}$ & $\begin{array}{l}\text { HEAVY- } \\
\text { IONS } \\
(\mathrm{MeV}- \\
\left.\mathrm{cm}^{2} / \mathrm{mg}\right)\end{array}$ & SEE \\
\hline NEC 4464 & $9 \pm 1$ & $11 \pm 3$ & Latchup \\
\hline IDT 6116 & 5.8 & $6.0 \pm 1.0$ & Upset \\
\hline AMD93L422 & 1.4 & 1.8 & Upset \\
\hline HC 6364 & 10 & $<18^{* *}$ & Upset \\
\hline
\end{tabular}

\section{TABLE 2. THRESHOLD LET}

** No measurements are available for LET below this value. No evidence of threshold at this value or above.

\section{Summary and Conclusions}

There is a linear relationship between the measured threshold LET ( $50 \%$ of the value at the beginning of the plateau) and the bias applied to the device. This relationship may not be linear for other devices, but the it should still be such that the threshold at reduced bias is related to the value at the operating bias such that changes in the threshold at operating bias will be reflected in changes at reduced bias. This suggest a procedure for monitoring SEU sensitivity at a QML manufacturing facility. Even rad-hard parts will become proton and neutron sensitive if the bias is sufficiently reduced.
A method for determining the thickness of the sensitive volume using SEU measurements at normal and grazing incidence has been developed. Values determined by this procedure agree with values obtained with charge collection measurements, SEM measurements, and estimates from the thickness of the epi-layer.

\section{References}

[1.] P.J. McNulty, "Predicting SEU Phenomena in Space", in 1990 IEEE Short Course: Microelectronics for the Natural Radiation Environments of Space (IEEE Nuclear and Radiation Effects Committee, 1990).

[2.] R.A. Reed, P.J. McNulty, W.J. Beauvais, and D.R. Roth, "Charge Collection Spectroscopy" this conference.

[3.] P.J. McNulty, W.J. Beauvais, R.A. Reed, D.R. Roth, E.G. Stassinopoulos, and E.G. Brucker, "Charge Collection at Large Angles of Incidence", IEEE Trans. Nucl. Sci. NS$\underline{39}, 1622-1629$ (1992)

[4.] P.J. McNulty, W.J. Beauvais, and D.R. Roth, "Determination of SEU Parameters of NMOS and CMOS SRAMS," IEEE Trans. Nucl. Sci. NS-38, 14631470 (1991)

[5.] W.J. Beauvais, P.J. McNulty, W.G. Abdel-Kader, and R.A. Reed, "SEU Parameters and Proton-Induced Upsets", to be presented at the RADECS Conference.

[6.] P.J. McNulty, D.R. Roth, W.J. Beauvais, W.G. AbdelKader, and D.C. Dinge, "Comparison of the Charge Collecting Properties of the Junctions and the SEU Response of Microelectronic Circuits" Int. J. Radiat. Instrum., Part D, Nucl. Tracks Radiat. Meas., 19, Nos. 14, 929-938 (1991).

[7.] E.L. Petersen, J.B. Langworthy, and S.E.Diehl, "Suggested Single Event Upset Figure of Merit", IEEE Trans. Nucl. Sci. NS-30, 4533-4539 (1983).

[8.] P.J. McNulty, W.G. Abdel-Kader, W.J. Beauvais, L. Adams, E.J. Daly, and R. Harboe-Sorensen, "Simple Model for proton Induced Latchup", this conference. 